\title{
All you need to know about gravitational waves
}

\author{
A beginner's guide to the BICEP2 discovery of primordial gravitational waves.
}

17 March 2014

Astronomers have announced the detection of the imprint of primordial 'gravitational waves' that originated in the Big Bang that created our Universe 13.8 billion years ago. The discovery has been hailed as a milestone in science, but the concepts involved will be unfamiliar to many people. Help is at hand. Here are some FAQs on gravitational waves and their answers.

\section{What is the significance of the BICEP2 announcement?}

Scientists will be unravelling the consequences of this discovery for years. But some major implications are already clear:

- Albert Einstein predicted 'gravitational waves' nearly 100 years ago, but he also calculated that they would be extremely feeble, so much so that he thought they would never be detected. BICEP2's findings are the most convincing evidence — short of direct detection — that gravitational waves actually exist.

- The waves are the confirmation of a cornerstone theory of the standard picture of cosmology. This theory,

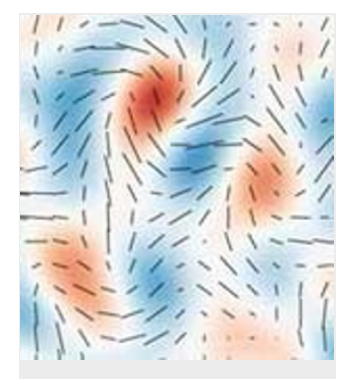


called inflation, says that during the first moments of its existence, the Universe underwent a brief period of exponential expansion.

Nature special: Waves from the Big Bang

- During inflation, the Universe's temperature — and thus the energies reached by elementary particles - were

trillions of times higher than can be achieved in any laboratory, even in particle accelerators such as the

Large Hadron Collider at CERN, near Geneva, Switzerland.

- Because inflation is a quantum phenomenon and gravitational waves are part of classical physics,

gravitational waves establish a link between the two, and could be the first evidence that gravity has a

quantum nature just like the other forces of nature (see 'How to see quantum gravity in Big Bang traces').

\section{What are gravitational waves?}

Gravity, according to Einstein's general theory of relativity, is how mass deforms the shape of space: near any massive body, the fabric of space becomes curved. But this curving does not always stay near the massive body. In particular, Einstein realized that the deformation can propagate throughout the Universe, just as seismic waves propagate in Earth's crust. Unlike seismic waves, however, gravitational waves can travel in empty space - and they do so at the speed of light.

If you could watch a gravitational wave head-on as it moves toward you, you would see it alternately stretching and compressing space, in the up-down and left-right directions.

\section{Is inflation the only thing that can produce gravitational waves?}

No. Anything that's massive and is undergoing violent acceleration is supposed to produce them. In practice, the only gravitational waves that we might be able to directly measure would be those from cataclysmic events such as two black holes colliding and fusing into one. Several observatories around the world are trying to pick up the distant noise of such black-hole mergers.

\section{Why couldn't gravitational waves be measured directly, but only detected via a radiotelescope?}

The gravitational waves that originated during inflation are still resonating throughout the Universe. But they are probably now too feeble to measure directly. Instead, scientists look for the imprint the waves have left in the broth of elementary particles that pervaded the Universe around 380,000 years after the Big Bang, which we see via the 'cosmic microwave background'. Observations of the microwave background radiation are made using telescopes that detect radio waves, and so the 'ripples' in the background caused by gravitational waves could only be detected by a radiotelescope.

\section{Why was the discovery made at the South Pole?}

The Amundsen-Scott South Pole Station, which hosts BICEP2, sits on the Antarctic ice sheet at more than 2,800 metres above sea level, so the atmosphere is thin. The air is also very dry, which is helpful as water vapour blocks microwaves. And Antarctica is also virtually uninhabited, so there is no interference from mobile phones, television broadcasts, and the rest of our electronic paraphernalia.

Nature I doi:10.1038/nature.2014.14886

Ron Cowen contributed reporting for this article. 